\title{
Threshold Improved QCD Corrections for Stop-Antistop production at Hadron colliders
}

\author{
U. Langenfeld \\ Humboldt-Universität zu Berlin \\ Newtonstraße 15, D-12489 Berlin, Germany \\ and \\ Julius-Maximilians-Universität Würzburg \\ Am Hubland, D-97074 Würzburg, Germany \\ ulangenfeldephysik.uni-wuerzburg.de
}

\begin{abstract}
I present improved predictions for the total hadronic cross section of stop-antistop production at hadron colliders including next-to-next-to-leading-order threshold corrections and approximated Coulomb corrections. The results are based on soft corrections, which are logarithmically enhanced near threshold. I present analytic formulas for the NNLO scaling functions at threshold and explicit numbers for the total hadronic cross sections for the Tevatron and the LHC. Finally I discuss the systematic error, the scale uncertainty and the PDF error of the hadronic cross section.
\end{abstract}




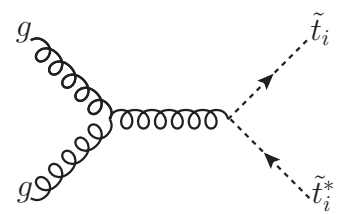

$a$
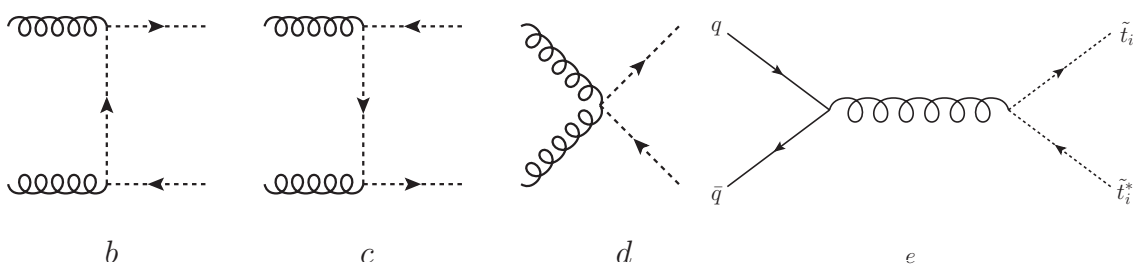

$d$

Figure 1: LO production of a $\tilde{t}_{i} \tilde{t}_{i}^{*}$ pair via $g g$ annihilation (diagrams a-d) and $q \bar{q}$ annihilation (diagram e).

\section{Introduction}

The Minimal Supersymmetric Standard model (MSSM) is an attractive extension [1, 2] of the very successful Standard Model. One property of this theory is its rich spectrum of new heavy particles which might be discovered at the LHC if they are lighter than $\approx 2 \mathrm{TeV}$. Searches for superymmetric particles are performed at the Tevatron and the LHC. No superpartners have been discovered so far. In the mSugra framework of the Minimal Supersymmetric Standard Model (MSSM), the lightest stop squark, one of the scalar supersymmetric partner particle of the top quark, is assumed to be the lightest supersymmetric coloured particle, lighter than the other scalar quarks. This is due to large nondiagonal elements in the stop mixing matrix, see f. e. the review [3]. The lightest stop squark might be the first coloured SUSY particle to be discovered. The cross section delivers information about the stop mass or, if the mass of the stop squark is roughly known from elsewhere, information about its spin [4]. If these particles cannot be discovered at the Tevatron or LHC, precise cross sections help to improve mass exclusion limits.

In this paper, I study the hadroproduction of stop-antistop-pairs

$$
p p / p \bar{p} \rightarrow \tilde{t}_{i} \tilde{t}_{i}^{*} X, \quad i=1,2,
$$

with its partonic subprocesses

$$
g g \rightarrow \tilde{t}_{i} \tilde{t}_{i}^{*} \text { and } q \bar{q} \rightarrow \tilde{t}_{i} \tilde{t}_{i}^{*}, q=u, d, c, s, b,
$$

including NNLO threshold contributions. The relevant leading order (LO) Feynman diagrams are shown in Fig. 1. The production of mixed stop pairs $\tilde{t}_{1} \tilde{t}_{2}^{*}$ or $\tilde{t}_{2} \tilde{t}_{1}^{*}$ starts at next to leading order (NLO) [5] and is therefore suppressed. This case will not be considered in this paper. The top parton density distribution in a proton is assumed to be zero, in contrast to the other quark parton density distributions. As a consequence, there is no gluino exchange diagram as for squark antisquark hadroproduction. For that reason, the $q \bar{q}$ channel is suppressed by a larger power of $\beta=\sqrt{1-4 m_{\tilde{t}}^{2} / s}$ due to $P$-wave annihilation. The final state must be in a state with angular momentum $l=1$ (denoted as $P$ ) to balance the spin of the gluon. Therefore, the case of stop-antistop hadroproduction needs a special treatment. At NLO, there is the $g q$ channel as an additional production mechanism. At the LHC with a center of mass energy of $7 \mathrm{TeV}$, one can expect for a luminosity of $1 \mathrm{fb}^{-1}, 100$ to $10^{4}$ events; even $10^{5}$ events are possible, if the stop is sufficiently light. At the LHC with a final center of mass energy of $14 \mathrm{TeV}$, even more events are expected to be collected. Hence it is necessary to predict the hadronic cross section with high accuracy.

So far, stop pairs have been searched for at the CDF [6-9] and D0 experiments [10,11] at the Tevatron using different strategies, for details see Tab. 1,

Squarks carry colour charge, so it is not surprising that processes involving Quantum Chromodynamics (QCD) obtain large higher-order corrections. For the production of colour-charged 
superymmetric particles, the NLO corrections have been calculated in Ref. [12], NLL and approximated NNLO corrections can be found in the Refs [13-16]. It has been found that these corrections are quite sizeable. Electroweak NLO corrections to stop-antistop production are discussed in Ref. [17, 18].

The theoretical aspects of $\tilde{t}_{i} \tilde{t}_{i}^{*}$ - production up to NLO have been discussed in Ref. [5] and of its NLL contributions in Ref. [19]. The hadronic LO and NLO cross section can be evaluated numerically using the programme Prospino [20].

In this paper, I calculate and study soft gluon effects to hadronic stop-antistop production in the framework of the $R$-parity- conserving MSSM. I use Sudakov resummation to generate the approximated NNLO corrections and include approximated two-loop Coulomb corrections and the exact scale dependence. I follow the approach for top-antitop production at the LHC and the Tevatron [21,22].

This paper is organised as follows. I review the LO and NLO contributions to the cross section. Then I describe the necessary steps to construct the approximated NNLO corrections. Using these results I calculate the approximated NNLO cross section and discuss the theoretical uncertainty due to scale variation and the error due to the parton density functions (PDFs). I give an example how these NNLO contributions reduce the scale uncertainty and improve exclusion limits.

\begin{tabular}{llcl}
\hline Ref. & Process & Exclusion limit & Assumptions or comments \\
\hline$[6]$ & $\tilde{t}_{1} \rightarrow c \tilde{\chi}_{1}^{0}$ & $m_{\tilde{t}_{1}}<100 \mathrm{GeV}$ & $m_{\chi_{1}^{0}}>50 \mathrm{GeV}$ \\
{$[10]$} & $p \bar{p} \rightarrow \tilde{t}_{1} \tilde{t}_{1}^{*}$ & $130 \mathrm{GeV}<m_{\tilde{t}_{1}}<190 \mathrm{GeV}$ & $\begin{array}{l}\text { Comparison of theor. predictions } \\
\text { with experimental and observed limits }\end{array}$ \\
& & & \\
{$[7,8]$} & $\tilde{t}_{1} \rightarrow b \tilde{\chi}_{1}^{ \pm} \rightarrow b \tilde{\chi}_{1}^{0} \ell^{ \pm} v_{\ell}$ & $128 \mathrm{GeV}<m_{\tilde{t}_{1}}<135 \mathrm{GeV}$ & \\
{$[9,11]$} & $\tilde{t}_{1} \rightarrow b \ell^{+} \tilde{v}_{\ell}$ & $m_{\tilde{t}_{1}}>180 \mathrm{GeV}$ & $m_{\tilde{v}} \geq 45 \mathrm{GeV}$ \\
& & $m_{\tilde{t}_{1}}=100 \mathrm{GeV}$ & $75 \mathrm{GeV} \leq m_{\tilde{v}} \leq 95 \mathrm{GeV}$ \\
\hline
\end{tabular}

Table 1: Exclusion limits for stop searches at the Tevatron.

\section{Theoretical Setup}

I focus on the inclusive hadronic cross section of hadroproduction of stop pairs, $\sigma_{p p \rightarrow \tilde{t}_{i} \tilde{t}_{i}^{*} X}$, which is a function of the hadronic center-of-mass energy $\sqrt{s}$, the stop mass $m_{\tilde{t}}$, the gluino mass $m_{\tilde{g}}$, the renormalisation scale $\mu_{r}$ and the factorisation scale $\mu_{f}$. In the standard factorisation approach of perturbative QCD, it reads

$$
\sigma_{p p / p \bar{p} \rightarrow \tilde{t} \tilde{t}^{*} X}\left(s, m_{\tilde{t}}^{2}, m_{\tilde{g}}^{2}, \mu_{f}^{2}, \mu_{r}^{2}\right)=\sum_{i, j=q, \bar{q}, g} \int_{4 m_{\tilde{t}}^{2}}^{s} d \hat{s} L_{i j}\left(\hat{s}, s, \mu_{f}^{2}\right) \hat{\sigma}_{i j \rightarrow \tilde{t} \tilde{t}^{*}}\left(\hat{s}, m_{\tilde{t}}^{2}, m_{\tilde{g}}^{2}, \mu_{f}^{2}, \mu_{r}^{2}\right)
$$

where the parton luminosities $L_{i j}$ are given as convolutions of the PDFs $f_{i / p}$ defined through

$$
L_{i j}\left(\hat{s}, s, \mu_{f}^{2}\right)=\frac{1}{s} \int_{\hat{s}}^{s} \frac{d z}{z} f_{i / p}\left(\mu_{f}^{2}, \frac{z}{s}\right) f_{j / p}\left(\mu_{f}^{2}, \frac{\hat{s}}{z}\right)
$$


Here, $\hat{s}$ denotes the partonic center of mass energy and $\mu_{f}^{2}, \mu_{r}^{2}$ are the factorisation and the renormalisation scale. The partonic cross section is expressed by dimensionless scaling function $f_{i j}^{(k l)}$

$$
\hat{\sigma}_{i j}=\frac{\alpha_{s}^{2}}{m_{\tilde{t}}^{2}}\left[f_{i j}^{(00)}+4 \pi \alpha_{s}\left(f_{i j}^{(10)}+f_{i j}^{(11)} L_{N}\right)+\left(4 \pi \alpha_{s}\right)^{2}\left(f_{i j}^{(20)}+f_{i j}^{(21)} L_{N}+f_{i j}^{(22)} L_{N}^{2}\right)\right]
$$

with $L_{N}=\ln \left(\frac{\mu^{2}}{m_{\tilde{t}}^{2}}\right)$. The LO scaling functions are given by [5]

$$
\begin{aligned}
f_{q \bar{q}}^{(00)} & =\frac{\pi}{54} \beta^{3} \rho=\frac{\pi}{54} \beta^{3}+\mathcal{O}\left(\beta^{5}\right), \\
f_{g g}^{(00)} & =\frac{\pi}{384} \rho\left[41 \beta-31 \beta^{3}+\left(17-18 \beta^{2}+\beta^{4}\right) \log \left(\frac{1-\beta}{1+\beta}\right)\right]=\frac{7 \pi}{384} \beta+\mathcal{O}\left(\beta^{3}\right) .
\end{aligned}
$$

Formulas for the higher orders of the $g g$-channel and its threshold expansions can be found in Refs [5, 14, 23], if one takes into account that in the case of stop-antistop production no sum over flavours and helicities is needed. $f_{g g}^{(10)}$ has been calculated numerically using Prospino [20]. A fit to this function for an easier numerical handling can be found in [14]. At NLO, $f_{q \bar{q}}^{(10)}$ is given at threshold by [5, 19]

$$
f_{q \bar{q}}^{(10)}=\frac{f_{q \bar{q}}^{(00)}}{4 \pi^{2}}\left(\frac{8}{3} \log ^{2}\left(8 \beta^{2}\right)-\frac{155}{9} \log \left(8 \beta^{2}\right)-\frac{\pi^{2}}{12 \beta}+54 \pi a_{1}^{q \bar{q}}\right) .
$$

The constant $a_{1}^{q \bar{q}}$ can be determined from a fit and is approximately given as $a_{1}^{q \bar{q}} \approx 0.042 \pm 0.001$. It depends mildly on the squark and gluino masses and on the stop mixing angle.

The $g q$-channel is absent at tree level. Its NLO contribution has been extracted from Prospino. This channel is strongly suppressed at threshold.

The $\ln \beta$ terms which appear in the threshold expansions of the NLO scaling functions can be resummed systematically to all orders in perturbation theory using the techniques described in [24-28]. Logarithmically enhanced terms for the hadronic production of heavy quarks admitting an $S$-wave are also studied in Ref. [29] for arbitrary $S U(3)_{\text {colour }}$ representations. The resummation is performed in Mellin space after introducing moments $N$ with respect to the variable $\rho=4 m_{\tilde{t}}^{2} / \hat{s}$ of the physical space:

$$
\hat{\sigma}\left(N, m_{\tilde{t}}^{2}\right)=\int_{0}^{1} d \rho \rho^{N-1} \hat{\sigma}\left(\hat{s}, m_{\tilde{t}}^{2}\right) .
$$

The resummed cross section is obtained for the individual color structures denoted as I from the exponential

$$
\frac{\hat{\sigma}_{i j, \mathbf{I}}\left(N, m_{\tilde{t}}^{2}\right)}{\hat{\sigma}_{i j, \mathbf{I}}^{B}\left(N, m_{\tilde{t}}^{2}\right)}=g_{i j, \mathbf{I}}^{0}\left(m_{\tilde{t}}^{2}\right) \cdot \exp \left[G_{i j, \mathbf{I}}(N+1)\right]+\mathcal{O}\left(N^{-1} \log ^{n} N\right),
$$

where all dependence on the renormalisation and factorisation scale $\mu_{r}$ and $\mu_{f}$ is suppressed and the respective Born term is denoted $\hat{\sigma}_{i j, \mathrm{I}}^{B}$. The exponent $G_{i j, \mathrm{I}}$ contains all large Sudakov logarithms $\log ^{k} N$ and the resummed cross section (10) is accurate up to terms which vanish as a power for large Mellin- $N$. To NNLL accuracy, $G_{i j, \mathbf{I}}$ is commonly written as

$$
G_{i j, \mathbf{I}}(N)=\log N \cdot g_{i j}^{1}(\lambda)+g_{i j, \mathbf{I}}^{2}(\lambda)+\frac{\alpha_{s}}{4 \pi} g_{i j, \mathbf{I}}^{3}(\lambda)+\ldots,
$$


where $\lambda=\beta_{0} \log N \alpha_{s} /(4 \pi)$. The exponential $\exp \left[G_{i j, \mathbf{I}}(N+1)\right]$ in Eq. (11) is independent from the Born cross section [21, 28]. The functions $g_{i j}^{k}, k=1,2,3$, for the octet color structure are explicitly given in Ref. [21] and can be taken over from the case of top-quark hadroproduction, the function $g_{q \bar{q}}^{0}$ is given by Eq. (36) in the App. A.2. All $g_{i j}^{k}, 0 \leq k \leq 3$, depend on a number of anomalous dimensions, i.e. the well-known cusp anomalous dimension $A_{q}$, the functions $D_{Q \bar{Q}}$ and $D_{q}$ controlling soft gluon emission, and the coefficients of the QCD $\beta$-function. The strength of soft gluon emission is proportional to the Casimir operator of the $S U(3)_{\text {colour }}$ representation of the produced state. This is identical for $t \bar{t}$ and $\tilde{t}_{i} \tilde{t}_{i}^{*}$-production. Expressions for $A_{q}$ and $D_{q}$ are given in the Refs. [30,31], and for $D_{Q \bar{Q}}$ in Ref. [32]. At higher orders, they also depend on the chosen renormalisation scheme, thus on the dynamical degrees of freedom.

For my fixed order NNLO calculation, I extracted the $\alpha_{s}^{2}$-terms from the right hand side of Eq. (10). At the end, I used Eqs (30) - (35) given in App. A.1 to convert the Mellin space result back to the physical $\rho$ space. I kept all those terms which are of the order $\beta^{3} \ln ^{k} \beta, 0 \leq k \leq 4$. Eventually, I end up with the following threshold expansion for $f_{q \bar{q}}^{(20)}$ :

$$
\begin{aligned}
& f_{q \bar{q}}^{(20)}=\frac{f_{q \bar{q}}^{(00)}}{\left(16 \pi^{2}\right)^{2}}\left[\frac{8192}{9} \ln ^{4} \beta+\left(-\frac{175616}{27}+\frac{16384}{3} \ln 2+\frac{1024}{27} n_{f}\right) \ln ^{3} \beta\right. \\
& +\left(\frac{525968}{27}-\frac{87808}{3} \ln 2-\frac{4480}{9} \pi^{2}+\frac{512}{3} C_{q \bar{q}}^{(1)}+12288 \ln ^{2} 2\right. \\
& \left.+\frac{512}{3} \ln 2 n_{f}-\frac{2080}{9} n_{f}-\frac{128}{9} \frac{\pi^{2}}{\beta}\right) \ln ^{2} \beta \\
& +\left(\frac{525968}{9} \ln 2-43904 \ln ^{2} 2+12288 \ln ^{3} 2-\frac{4960}{9} C_{q \bar{q}}^{(1)}-\frac{2980288}{81}\right. \\
& +\frac{61376}{9} \zeta_{3}+\frac{49280}{27} \pi^{2}-\frac{4480}{3} \pi^{2} \ln 2+512 C_{q \bar{q}}^{(1)} \ln 2-2 D_{Q \bar{Q}}^{(2)} \\
& +\left(-\frac{128}{9} \pi^{2}+256 \ln ^{2} 2+\frac{45568}{81}-\frac{2080}{3} \ln 2\right) n_{f} \\
& \left.+\left(\frac{266}{9}-\frac{128}{9} \ln 2-\frac{4}{9} n_{f}\right) \frac{\pi^{2}}{\beta}\right) \ln \beta \\
& \left.+\left(-\frac{13}{3}+\frac{22}{3} \ln 2+\left(\frac{10}{27}-\frac{4}{9} \ln 2\right) n_{f}\right) \frac{\pi^{2}}{\beta}+\frac{1}{27} \frac{\pi^{4}}{\beta^{2}}+C_{q \bar{q}}^{(2)}\right] .
\end{aligned}
$$

$C_{q \bar{q}}^{(1)}$ is given as $C_{q \bar{q}}^{(1)}=216 \pi a_{1}^{q \bar{q}}-\frac{310}{27}, C_{q \bar{q}}^{(2)}$ is the unknown 2-loop matching constant, which is set to zero in the numerical evaluation and $D_{Q \bar{Q}}^{(2)}=460-12 \pi^{2}+72 \zeta_{3}-\frac{88}{3} n_{f}$, see Ref. [32]. The $\beta^{3}$-behaviour of the threshold expansion of the LO cross section comes only from the $P$-wave of the final state $\tilde{t}_{i} \tilde{t}_{i}^{*}$ as mentioned in the introduction and does not spoil the factorisation properties in the threshold region of the phase space. Note that the formulas given in Ref. [28] can easily extended to Mellin transformed cross sections $\omega$, which vanish as a power $\beta^{k}$ with $k \geq 1$. These two more powers of $\beta$ in the $q \bar{q}$-channel of $\tilde{t}_{i} \tilde{t}_{i}^{*}$ - production lead to an additional $1 / N$ factor in Mellin space. Eq. (10) reproduces the known NLO threshold expansion given in Ref. [5, 19] for the $q \bar{q}$ channel. This is a check that the approach works. Logarithmically enhanced terms which are suppressed by an additional $1 / N$ factor appear in the resummation of the (sub)leading 
$\log ^{k}(N) / N$-terms of the corrections for the structure function $F_{L}$ and are studied in detail in the Refs [33, 34]. For these reasons I apply the formulas derived for heavy quark hadroproduction.

The coefficients of the $\ln ^{4} \beta, \ln ^{3} \beta$, and $\ln ^{2} \beta$ terms depend only on first order anomalous dimensions and on the constant $C_{q \bar{q}}^{(1)}$ which is related to the NLO constant $a_{1}^{q \bar{q}}$, see the equation above. The linear $\log \beta$ term depends on $C_{q \bar{q}}^{(1)}$ as well and on other first order (NLO) contributions, but also on second-order anomalous dimensions and non-Coulomb potential contributions [29]. In Tab. 2, I show for four examples how these parts contribute to the hadronic NNLO threshold corrections. The numbers show that terms which have an NLO origin contribute most and that NNLO contributions have a small but sizeable effect.

I also included the Coulomb corrections up to NNLO. For the singlet case, the Coulomb contributions are studied in Ref. [35]. Generalisation to other colour structures requires the substitution of the corresponding group factors and decomposition of the colour structures of the considered process in irreducible colour representations. The last step will not be necessary for stop pair production in the $q \bar{q}$-annihilation channel. The NLO Coulomb corrections agree with the NLO Coulomb corrections for top antitop production [5.14]. In both cases, only the colour octet contributes to the scaling function at the corresponding leading order in $\beta$. Therefore, I have used as an approximation for the NNLO Coulomb contributions for $\tilde{t} \tilde{t}^{*}$-production the same NNLO Coulomb contributions as for $t \bar{t}$-production [14,21, 35]. Gauge invariance together with Supersymmetry support this approximation. Note that the $\log ^{2} \beta / \beta$-term comes from interference of the NLO Coulomb-contribution with the NLO threshold logarithms. Tab. 2 shows also the NLO and the pure NNLO Coulomb contributions to the NNLO threshold corrections at the hadronic level.

The scale-dependent scaling functions are derived by renormalisation group techniques following Refs [36, 37]:

$$
\begin{aligned}
& f_{i j}^{(11)}= \frac{1}{16 \pi^{2}}\left(2 \beta_{0} f_{i j}^{(00)}-f_{k j}^{(00)} \otimes P_{k i}^{(0)}-f_{i k}^{(00)} \otimes P_{k j}^{(0)}\right), \\
& f_{i j}^{(21)}= \frac{1}{\left(16 \pi^{2}\right)^{2}}\left(2 \beta_{1} f_{i j}^{(00)}-f_{k j}^{(00)} \otimes P_{k i}^{(1)}-f_{i k}^{(00)} \otimes P_{k j}^{(1)}\right) \\
&+\frac{1}{16 \pi^{2}}\left(3 \beta_{0} f_{i j}^{(10)}-f_{k j}^{(10)} \otimes P_{k i}^{(0)}-f_{i k}^{(10)} \otimes P_{k j}^{(0)}\right), \\
& f_{i j}^{(22)=} \frac{1}{\left(16 \pi^{2}\right)^{2}}\left(f_{k l}^{(00)} \otimes P_{k i}^{(0)} \otimes P_{l j}^{(0)}+\frac{1}{2} f_{i n}^{(00)} \otimes P_{n l}^{(0)} \otimes P_{l j}^{(0)}+\frac{1}{2} f_{n j}^{(00)} \otimes P_{n k}^{(0)} \otimes P_{k i}^{(0)}\right. \\
&\left.\quad+3 \beta_{0}^{2} f_{i j}^{(00)}-\frac{5}{2} \beta_{0} f_{i k}^{(00)} \otimes P_{k j}^{(0)}-\frac{5}{2} \beta_{0} f_{k j}^{(00)} \otimes P_{k i}^{(0)}\right),
\end{aligned}
$$

where $\otimes$ denotes the standard Mellin convolution; these are ordinary products in Mellin space using Eq. (9). Repeated indices imply summation over admissible partons. However, I restrict myself for phenomenological applications to the numerically dominant diagonal parton channels at two-loop. Note that the scale dependence is exact at all energies, even away from threshold, because the Eqs (13)-(15) depend on functions which are at least one order lower than they themselves have. The functions $P_{i j}(x)$ are called splitting functions and govern the PDF evolution. They have the expansion

$$
P_{i j}(x)=\frac{\alpha_{s}}{4 \pi} P_{i j}^{(0)}(x)+\left(\frac{\alpha_{s}}{4 \pi}\right)^{2} P_{i j}^{(1)}(x)+\ldots
$$

Explicit expressions for the $P_{i j}^{(k)}$ can be found in Refs [30, 38]. 

fies to

Analytical results for $f_{g g}^{(11)}$ and $f_{q \bar{q}}^{(11)}$ are given in Ref. [23]. For the $g q$-channel, Eq. (13) simpli-

$$
f_{g q}^{(11)}=-\frac{1}{16 \pi^{2}}\left(P_{g q}^{(0)} \otimes f_{g g}^{(0)}+\frac{1}{2 n_{f}} P_{q g}^{(0)} \otimes f_{q \bar{q}}^{(0)}\right) .
$$

The integration can be done explicitly yielding

$$
\begin{aligned}
f_{g q}^{(11)}=\frac{1}{51840 \pi}[\beta & \left(-176-1083 \rho+1409 \rho^{2}\right)+15 \rho\left(26-(27-24 \ln 2) \rho-4 \rho^{2}\right) L_{2} \\
& \left.+180 \rho^{2}\left(2 L_{4}-L_{6}\right)\right]
\end{aligned}
$$

where the functions $L_{2}, L_{4}$, and $L_{6}[23]$ are defined as

$$
L_{2}=\log \left(\frac{1+\beta}{1-\beta}\right), \quad L_{4}=\mathrm{Li}_{2}\left(\frac{1-\beta}{2}\right)-\mathrm{Li}_{2}\left(\frac{1+\beta}{2}\right), L_{6}=\log ^{2}(1-\beta)-\log ^{2}(1+\beta) .
$$

The high energy limit of this scaling function is

$$
\lim _{\beta \rightarrow 1} f_{g q}^{(11)}=-\frac{11}{3240 \pi}
$$

which agrees with the result given in Ref. [5].

The threshold expansions of the NNLO-scale-dependent scaling functions of the $q \bar{q}$ channel read

$$
\begin{aligned}
f_{q \bar{q}}^{(21)}=- & \frac{f_{q \bar{q}}^{(00)}}{\left(16 \pi^{2}\right)^{2}}\left[\frac{8192}{9} \ln ^{3} \beta+\left(\frac{256}{3} n_{f}+\frac{32768}{9} \ln 2-\frac{46976}{9}\right) \ln ^{2} \beta\right. \\
& +\left(-\frac{383104}{27} \ln 2+\frac{798872}{81}+\frac{14336}{3} \ln ^{2} 2-\frac{8080}{27} n_{f}-\frac{2240}{9} \pi^{2}-\frac{64}{9} \frac{\pi^{2}}{\beta}\right. \\
& \left.+\frac{256}{3} C^{(1)}+256 n_{f} \ln 2\right) \ln \beta+\frac{4540}{81} n_{f}-\frac{1924}{9} C^{(1)}+2048 \ln ^{3} 2 \\
& +\frac{393004}{27} \ln 2-\frac{1449488}{243}+8 C^{(1)} n_{f}-\frac{85856}{9} \ln ^{2} 2+\frac{14240}{9} \zeta_{3} \\
& +\frac{11024}{27} \pi^{2}-\frac{1088}{3} \pi^{2} \ln 2+192 \ln ^{2} 2 n_{f}+\frac{25}{3} \frac{\pi^{2}}{\beta}+\frac{256}{3} C^{(1)} \ln 2 \\
& \left.-\frac{11800}{27} n_{f} \ln 2-\frac{32}{27} \pi^{2} n_{f}-\frac{2}{3} n_{f} \frac{\pi^{2}}{\beta}\right], \\
f_{q \bar{q}}^{(22)}= & \frac{f_{q \bar{q}}^{(00)}}{\left(16 \pi^{2}\right)^{2}}\left[\frac{2048}{9} \ln ^{2} \beta+\left(-\frac{27616}{27}+\frac{320}{9} n_{f}+\frac{4096}{9} \ln 2\right) \ln \beta\right. \\
& -\frac{2108}{27} n_{f}+\frac{112351}{81}-\frac{27616}{27} \ln 2+\frac{2048}{9} \ln 2+\frac{320}{9} n_{f} \ln 2 \\
& \left.-\frac{256}{9} \pi^{2}+\frac{4}{3} n_{f}^{2}\right]
\end{aligned}
$$

with $C^{(1)}=54 \pi a_{1}^{q \bar{q}}$. 


\begin{tabular}{|c|c|c|c|c|c|c|c|c|c|c|}
\hline \multirow[t]{2}{*}{ Collider } & \multirow[t]{2}{*}{$\overline{m_{\tilde{t}}}$} & \multirow[t]{2}{*}{$\sum$} & \multirow[t]{2}{*}{$\ln ^{4}(\beta)$} & \multirow[t]{2}{*}{$\ln ^{3}(\beta)$} & \multirow[t]{2}{*}{$\ln ^{2}(\beta)$} & \multicolumn{3}{|c|}{$\log (\beta)$} & \multirow[t]{2}{*}{$\mathrm{C}_{\mathrm{NLO}}$} & \multirow[t]{2}{*}{$\mathrm{C}_{\mathrm{NNLO}}$} \\
\hline & & & & & & $\mathrm{nC}$ & $D_{Q \bar{Q}}^{(2)}$ & rest & & \\
\hline \multirow[t]{2}{*}{ LHC $14 \mathrm{TeV}$} & $300 \mathrm{GeV}$ & 74.54 & 5.33 & 18.80 & 31.87 & & 27.71 & & -9.45 & 0.29 \\
\hline & & & & & & -2.23 & 17.90 & 12.04 & & \\
\hline \multirow[t]{2}{*}{ LHC $14 \mathrm{TeV}$} & $600 \mathrm{GeV}$ & 2.93 & 0.24 & 0.81 & 1.28 & & 0.97 & & -0.37 & -0.01 \\
\hline & & & & & & -0.08 & 0.63 & 0.42 & & \\
\hline \multirow[t]{2}{*}{ LHC 7 TeV } & $300 \mathrm{GeV}$ & 17.5 & 1.42 & 4.83 & 7.66 & & 5.85 & & -2.21 & -0.06 \\
\hline & & & & & & -0.47 & 3,78 & 2.54 & & \\
\hline \multirow[t]{2}{*}{ Tevatron } & $300 \mathrm{GeV}$ & 1.41 & 0.16 & 0.48 & 0.62 & & 0.34 & & -0.17 & -0.02 \\
\hline & & & & & & -0.03 & 0.22 & 0.15 & & \\
\hline
\end{tabular}

Table 2: Individual hadronic contributions of the log-powers and Coulomb-corrections to the NNLO threshold contributions of the $q \bar{q}$-channel in $\mathrm{fb} \cdot \ln ^{4}(\beta)$ has to be understood as $\frac{f_{q \bar{q}}^{(00)}}{\left(16 \pi^{2}\right)^{2}} \cdot \frac{8192}{9} \ln ^{4}(\beta)$, analogously for the other terms. The linear log-term is decomposed into contributions coming from nonCoulomb potential terms, from the two loop anomalous dimension $D_{Q_{\bar{Q}^{\prime}}}^{(2)}$ and, finally, the rest. The Coulomb contribution are decomposed into contributions coming from the interference of NLO threshold logarithms with NLO Coulomb corrections $\mathrm{C}_{\mathrm{NLO}}$ and pure NNLO Coulomb corrections $\mathrm{C}_{\mathrm{NNLO}} . \sum$ denotes the sum over all NNLO threshold contributions. The PDF set used is MSTW 2008 NNLO [39].

In Fig. 2, I show the LO, NLO, and NNLO scaling functions. The scaling functions $f_{q \bar{q}}^{(00)}, f_{q \bar{q}}^{(11)}$ $f_{q \bar{q}}^{(20)}$, and $f_{q \bar{q}}^{(22)}$ depend only on the dimensionless variable $\eta=\frac{\hat{s}}{4 m_{\bar{f}}^{2}}-1$, but $f_{q \bar{q}}^{(10)}$ and $f_{q \bar{q}}^{(21)}$ depend also mildly on the masses of the squarks and the gluino and the stop mixing angle [5]. At the hadronic level, the effect for the NLO + NLL cross section is smaller than $2 \%$ [19], so I neglect them.

As example point, I have chosen the following masses: $m_{\tilde{t}_{1}}=300 \mathrm{GeV}, m_{\tilde{q}}=400 \mathrm{GeV}=$ $1.33 m_{\tilde{t}_{1}}, m_{\tilde{t}_{2}}=480 \mathrm{GeV}=1.6 m_{\tilde{t}_{1}}, m_{\tilde{g}}=500 \mathrm{GeV}=1.67 m_{\tilde{t}_{1}}$, and $\theta=\pi / 2$, i.e. $m_{\tilde{t}_{1}}=m_{\tilde{t}_{R}}$ and $m_{\tilde{t}_{2}}=m_{\tilde{t}_{\mathrm{L}}}$. When varying the stop mass I conserve these mass relations. I restrict myself to the lighter stop, but the results also apply to the heavier stop, because the gluon-stop-stop interactions entering my process do not distinguish between the left-handed and the right-handed stop squarks.

\section{Results}

\subsection{Hadronic cross section}

I start with the discussion of the total hadronic cross section, which is obtained by convoluting the partonic cross section with the PDFs, see Eq. (3). I keep the $g g$ and the $q \bar{q}$ channel at all orders up to NNLO, and for the scale-dependent terms, only contributions coming from diagonal parton channels are considered. Only the NLO contributions of the $g q$ channel are considered, which are the leading contributions of this channel. The scale dependence of this channel is given by Eq. (17).

I define the NLO and NNLO $K$ factors as

$$
K_{\mathrm{NLO}}=\frac{\sigma^{\mathrm{NLO}}}{\sigma^{\mathrm{LO}}}, \quad K_{\mathrm{NNLO}}=\frac{\sigma^{\mathrm{NNLO}}}{\sigma^{\mathrm{NLO}}} .
$$



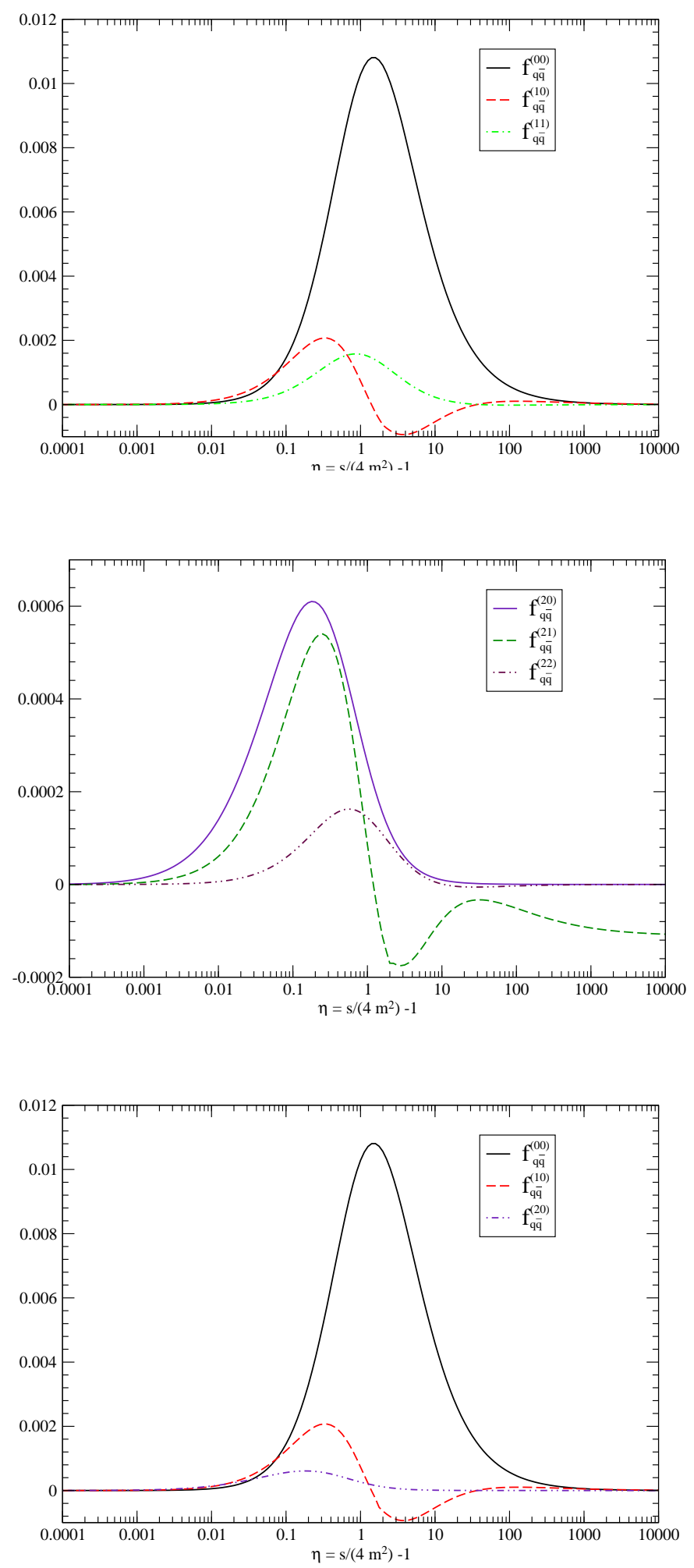

Figure 2: Scaling functions $f_{q \bar{q}}^{(i j)}$ with $i=0,1,2$ and $j \leq i$. The masses are $m_{\tilde{t}_{1}}=300 \mathrm{GeV}, m_{\tilde{q}}=400 \mathrm{GeV}$, $m_{\tilde{t}_{2}}=480 \mathrm{GeV} m_{\tilde{g}}=500 \mathrm{GeV}$. 
I use for all orders MSTW2008 NNLO PDFs [39], if not otherwise stated. Therefore, the $K$ factors account only for the pure higher order corrections of the partonic cross section (convoluted with the PDFs) and not for higher order corrections of the PDFs and the strong coupling constant $\alpha_{s}$.

In the left column of Fig. 3. I show the total hadronic cross section for the LHC (7 TeV first row, $14 \mathrm{TeV}$ second row) and the Tevatron (third row) as a function of the stop mass. Similar to top-antitop [21,22] and squark-antisquark production [14], the total cross section shows a strong mass dependence. At the LHC $(14 \mathrm{TeV})$, the cross section decreases within the shown stop mass range from about $1000 \mathrm{pb}$ to $10^{-2} \mathrm{pb}$. In the right column of Fig. 3 , I show the corresponding $\mathrm{K}$ factors.

For example, for a stop mass of $300 \mathrm{GeV}$ produced at the $\mathrm{LHC}$ with $14 \mathrm{TeV}$, I have a total cross section of $6.57 \mathrm{pb}, 9.96 \mathrm{pb}, 10.92 \mathrm{pb}$ at LO, NLO, NNLO approx, respectively. For a stop mass of $600 \mathrm{GeV}$, I find $0.146 \mathrm{pb}, 0.216 \mathrm{pb}, 0.244 \mathrm{pb}$. The $K$-factors are $K_{\mathrm{NLO}} \approx 1.5$ and $K_{\mathrm{NNLO}} \approx 1.1$ and 1.13. For the $\mathrm{LHC}$ at $7 \mathrm{TeV}$, one finds similar values for stop masses in the interval $100 \mathrm{GeV} \leq$ $m_{\tilde{t}} \leq 600 \mathrm{GeV}$. At the Tevatron, I have $K_{\mathrm{NLO}}=1.3 \ldots 1.4$ and $K_{\mathrm{NNLO}} \approx 1.2$ for stop masses in the range $100 \mathrm{GeV} \leq m_{\tilde{t}} \leq 300 \mathrm{GeV}$.

In Tabs 3 - 5, see App. B, values for the total hadronic cross section for different masses, PDF sets, scales and colliders are shown. The values for the PDF sets Cteq6.6 [40], MSTW 2008 NNLO [39], and CT10 [41] show only small differences, whereas the ABKM09 NNLO (5 flavours) PDFs differ in the treatment of the gluon PDF from the other PDF sets. This leads to sizeable differences in the total cross sections.

\subsection{Theoretical and Systematic Uncertainty and PDF error}

In this section, I address the following sources for errors: the systematic theoretical error, the scale uncertainty, and the PDF error.

In Tab. 2, I listed the individual $\ln ^{k} \beta$ contributions to the total NNLO contributions. Note that the NNLO matching constants $C_{i j}^{(2)}$ are unknown and set to zero. Compared to the total NNLO contributions the $\ln ^{1} \beta$ term is quite sizeable, this translates to a roughly $3-5 \%$ contribution to the NNLO cross section. To estimate the systematic error coming from the NNLO matching constants $C_{i j}^{(2)}$, I proceed as described in Ref. [22]. I find for the ratio $\sigma_{\mathrm{NLL}+\mathrm{Coul}} / \sigma_{\text {exact }}=1.10 \ldots 1.25$. This ratio translates to an estimate for the relative systematic error coming from the NNLO matching constants as $1-2.5 \%$.

The total hadronic LO, NLO, and NNLO cross sections are shown on the left of Fig. 4 as a function of the stop mass and for variations of the scale $\mu$ with $m_{\tilde{t}} / 2 \leq \mu \leq 2 m_{\tilde{t}}$, where I have identified the factorisation scale with the renormalisation scale. The width of the band indicates the scale uncertainty, which becomes smaller when going from LO to NLO and NNLO. On the right-hand side of Fig. 4, the scale dependence for the example point is shown in more detail. I quote as theoretical uncertainty

$$
\min \sigma(\mu) \leq \sigma\left(m_{\tilde{t}}\right) \leq \max \sigma(\mu),
$$

where the min and max are to be taken over the interval $\left[m_{\tilde{t}_{1}} / 2,2 m_{\tilde{t}_{1}}\right]$. At LO and NLO, the minimal value is taken at $\mu=2 m_{\tilde{t}}$ and the maximal value at $\mu=m_{\tilde{t}} / 2$. However, this is not longer true at NNLO. For the theoretical error, one finds

$$
\sigma_{\mathrm{LO}}=6.57_{-1.43}^{+2.06} \mathrm{pb}, \quad \sigma_{\mathrm{NLO}}=9.96_{-1.22}^{+1.17} \mathrm{pb}, \quad \sigma_{\mathrm{NNLO}}=10.90_{-0.18}^{+0.01} \mathrm{pb} .
$$

As one can see, there is a strong scale dependence at LO, becoming weaker at NLO, and is 

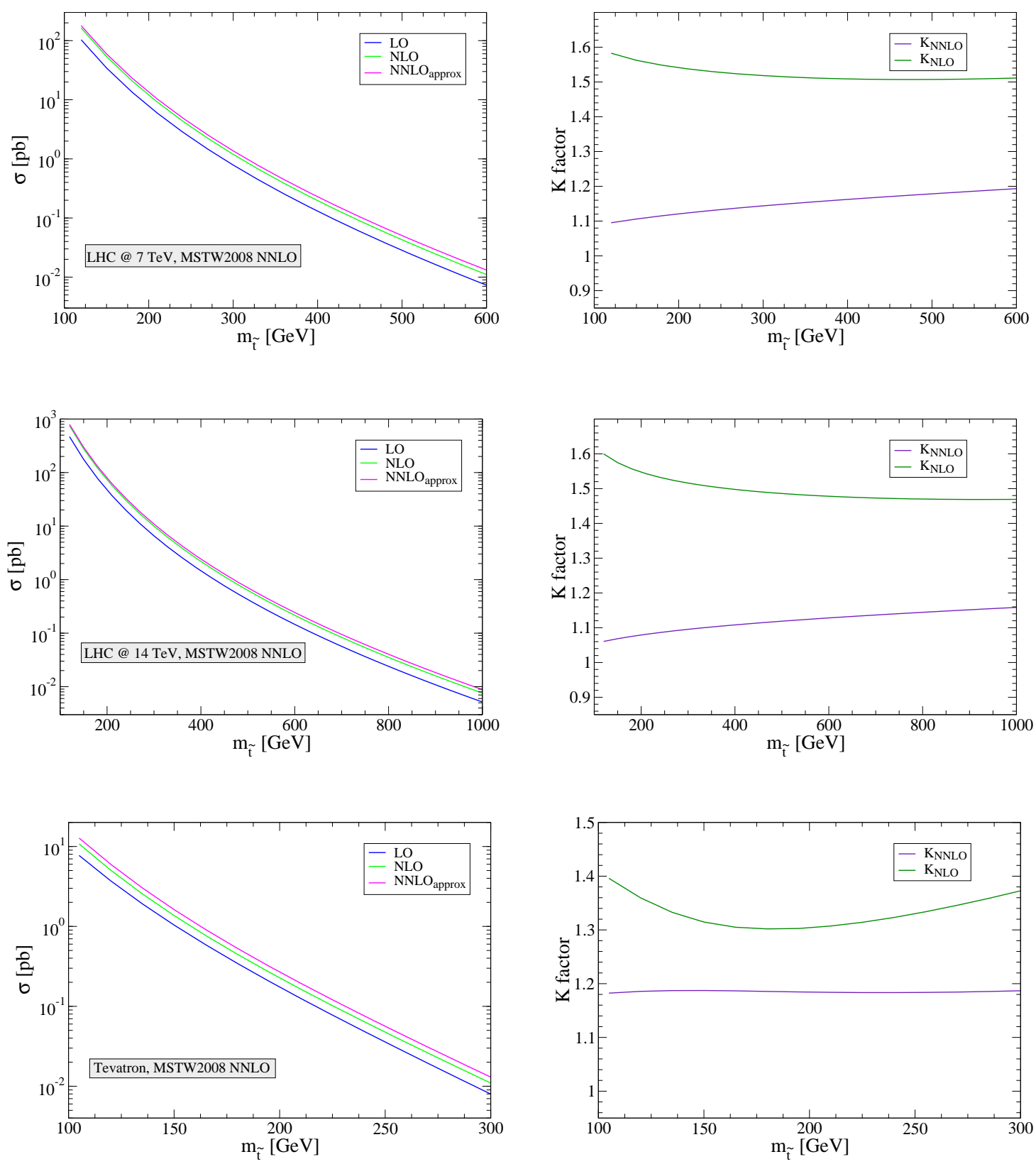

Figure 3: Total hadronic cross section at LO, NLO, and $\mathrm{NNLO}_{\text {approx }}$ at the $\mathrm{LHC} 7 \mathrm{TeV}$ (first row) and $14 \mathrm{TeV}$ (second row) and the Tevatron (1.96 TeV, third row). The right column shows the corresponding $\mathrm{K}$ factors. The PDF set used is MSTW2008 NNLO [39].

flattend out at NNLO within the considered range. This flattening gives a hint that the approach is reliable.

Using renormalisation group techniques, one recovers the full dependence on the renormalisation scale $\mu_{r}$ and factorisation scale $\mu_{f}$. I have done this for the example point for the NLO and the NNLO cross section, see Fig. 5. I define the theoretical uncertainty coming from an 

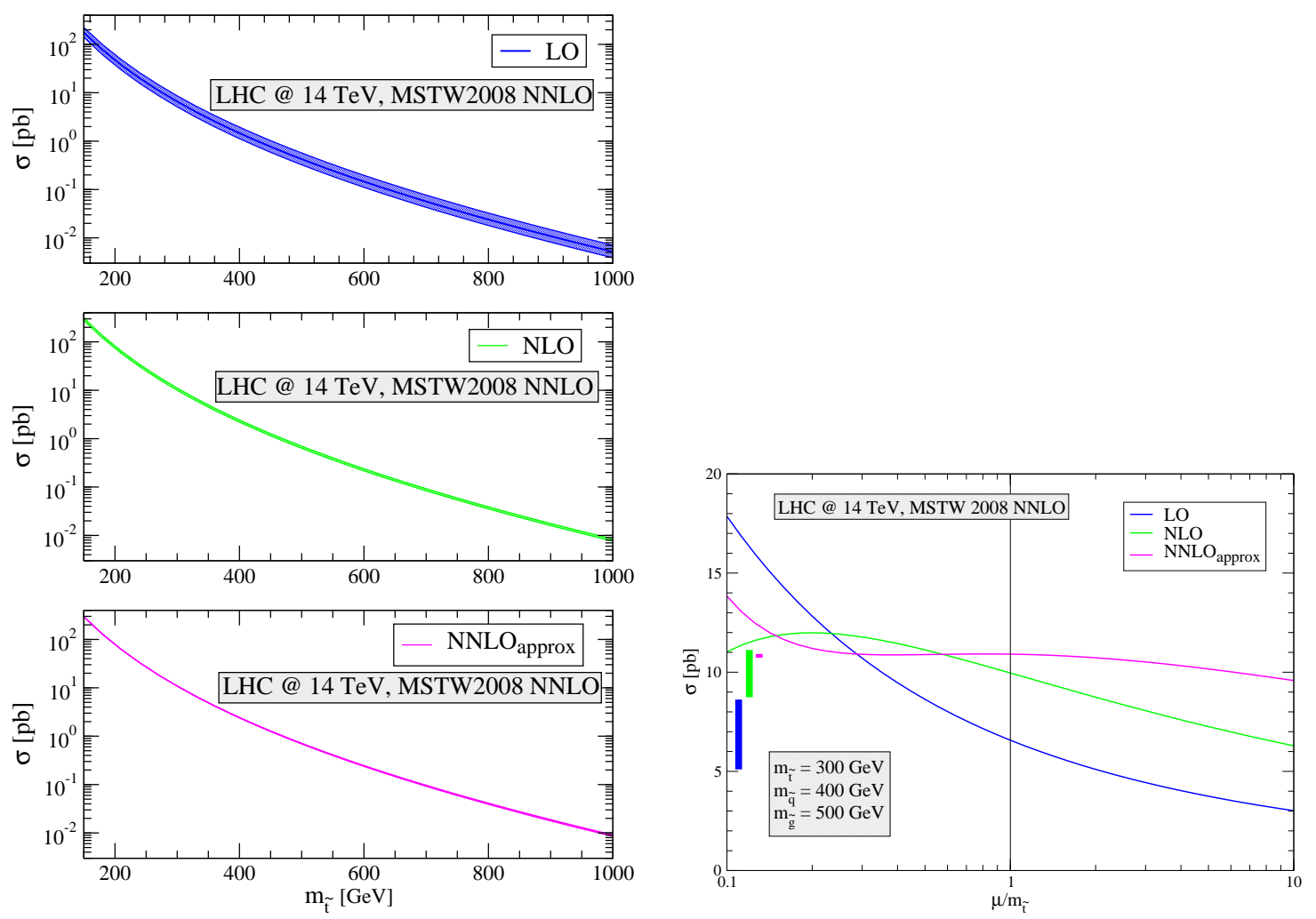

Figure 4: Left hand side: Theoretical uncertainty of the total hadronic cross section at the LHC (14 TeV) at LO (upper figure, blue band), NLO (central figure, green band), $\mathrm{NNLO}_{\text {approx }}$ (lower figure, purple line). At $\mathrm{NNLO}_{\text {approx, }}$ the theoretical uncertainty has shrunk to a small band. Right hand side: Scale dependence of the total hadronic cross section for the example point $m_{\tilde{t}_{1}}=300 \mathrm{GeV}, m_{\tilde{q}}=400 \mathrm{GeV}, m_{\tilde{t}_{2}}=480 \mathrm{GeV}$, $m_{\tilde{g}}=500 \mathrm{GeV}$. The vertical bars indicate the total scale variation in the range $\left[m_{\tilde{t}} / 2,2 m_{\tilde{t}}\right]$.

independent variation of $\mu_{r}$ and $\mu_{f}$ in the standard range $\mu_{r}, \mu_{f} \in\left[m_{\tilde{t}} / 2,2 m_{\tilde{t}}\right]$ as

$$
\min \sigma\left(\mu_{r}, \mu_{f}\right) \leq \sigma\left(m_{\tilde{t}}\right) \leq \max \sigma\left(\mu_{r}, \mu_{f}\right)
$$

The contour lines of the total cross section for the example point with an independent variation of $\mu_{r}$ and $\mu_{f}$ are shown in Fig. 5. Note that the range of the axes is from $\log _{2}\left(\mu_{r, f} / m_{\tilde{t}_{1}}\right)=-1$ to $\log _{2}\left(\mu_{r, f} / m_{\tilde{t}_{1}}\right)=1$. The scale variation with fixed scales $\mu_{r}=\mu_{f}$ proceeds along the diagonal from the lower left to the upper right corner of the figure. The gradient of the NLO contour lines lies approximately in the $\mu_{r}=\mu_{f}$ direction, meaning that the theoretical error from the definition in Eq. (26) is the same as if one sets $\mu_{r}=\mu_{f}$. For the NNLO case one observes the opposite situation: the contour lines are nearly parallel to the diagonal $\mu_{r}=\mu_{f}$. I obtain a larger uncertainty in that case:

$$
\sigma_{\mathrm{NLO}}=9.96_{-1.22}^{+1.17} \mathrm{pb}, \quad \sigma_{\mathrm{NNLO}}=10.90_{-0.46}^{+1.05} \mathrm{pb} .
$$

Another source of error to discuss is the PDF error. I calculated the PDF uncertainty according to Ref. [40] for the two PDF sets CT10 and MSTW2008 NNLO (90\% C.L.). In both cases, the uncertainty increases with higher stop masses due to large uncertainties of the gluon PDF in high $x$-ranges. For CT10, I find as relative errors $\approx 3 \%$ for $m_{\tilde{t}}=100 \mathrm{GeV}$ and $\approx 18 \%$ for $m_{\tilde{t}}=$ $1000 \mathrm{GeV}$, and for MSTW2008 NNLO, the relative errors are $\approx 3 \%$ for $m_{\tilde{t}}=100 \mathrm{GeV}$ and $\approx 10 \%$ for $m_{\tilde{t}}=1000 \mathrm{GeV}$. The relative error of the MSTW2008 NNLO PDF set is smaller for large stop masses compared to the CT10 PDFs. 

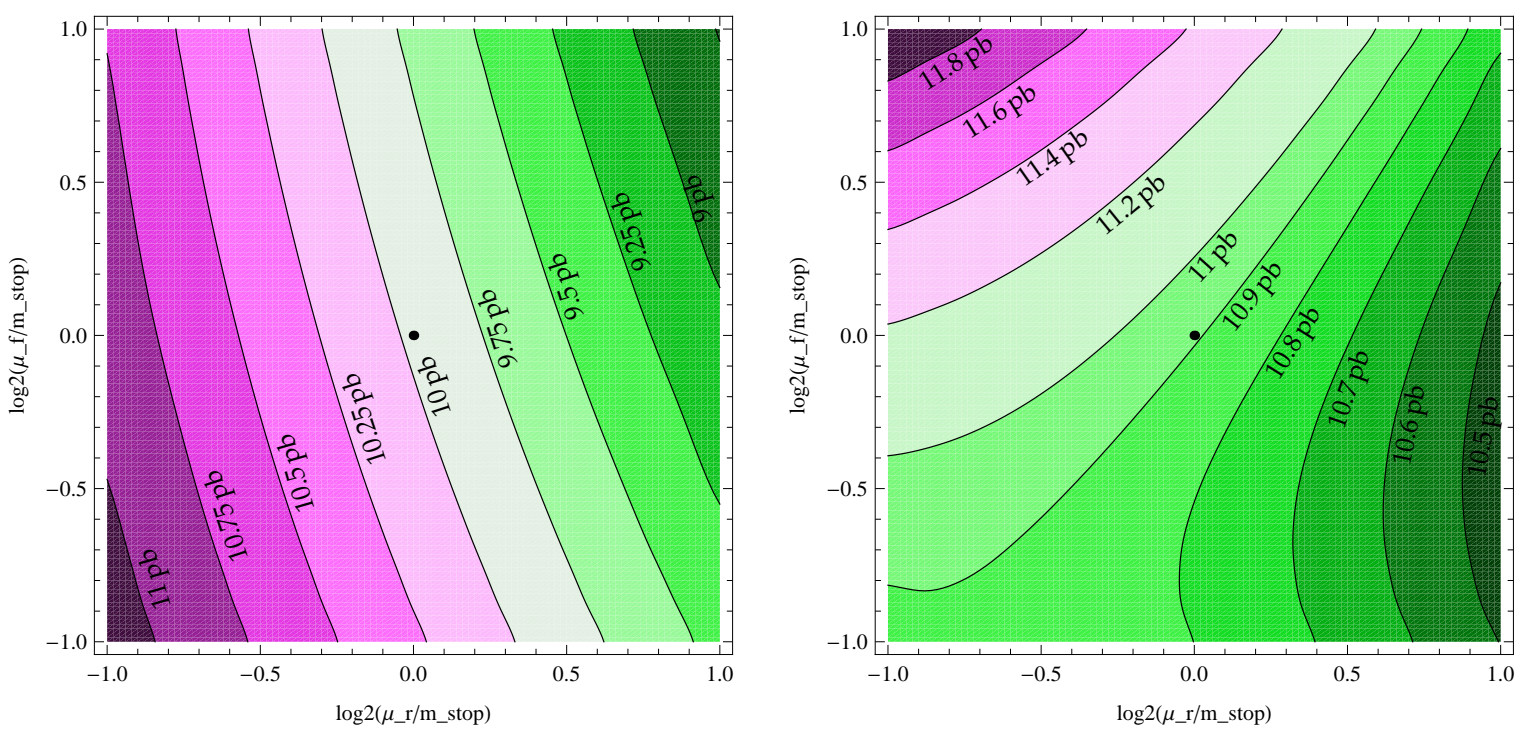

Figure 5: Contour lines of the total hadronic NLO (left) and NNLO (right) cross section from the independent variation of the renormalisation and factorisation scale $\mu_{r}$ and $\mu_{f}$ for LHC, $14 \mathrm{TeV}$, with PDF set MSTW2008 NNLO [39] for the example point with $m_{\tilde{t}}=300 \mathrm{GeV}$. The dot in the middle of the figure indicates the cross section for $\mu_{r}=\mu_{f}=m_{\tilde{t}}$, and the range corresponds to $\mu_{f}, \mu_{r} \in\left[m_{\tilde{t}} / 2,2 m_{\tilde{t}}\right]$.
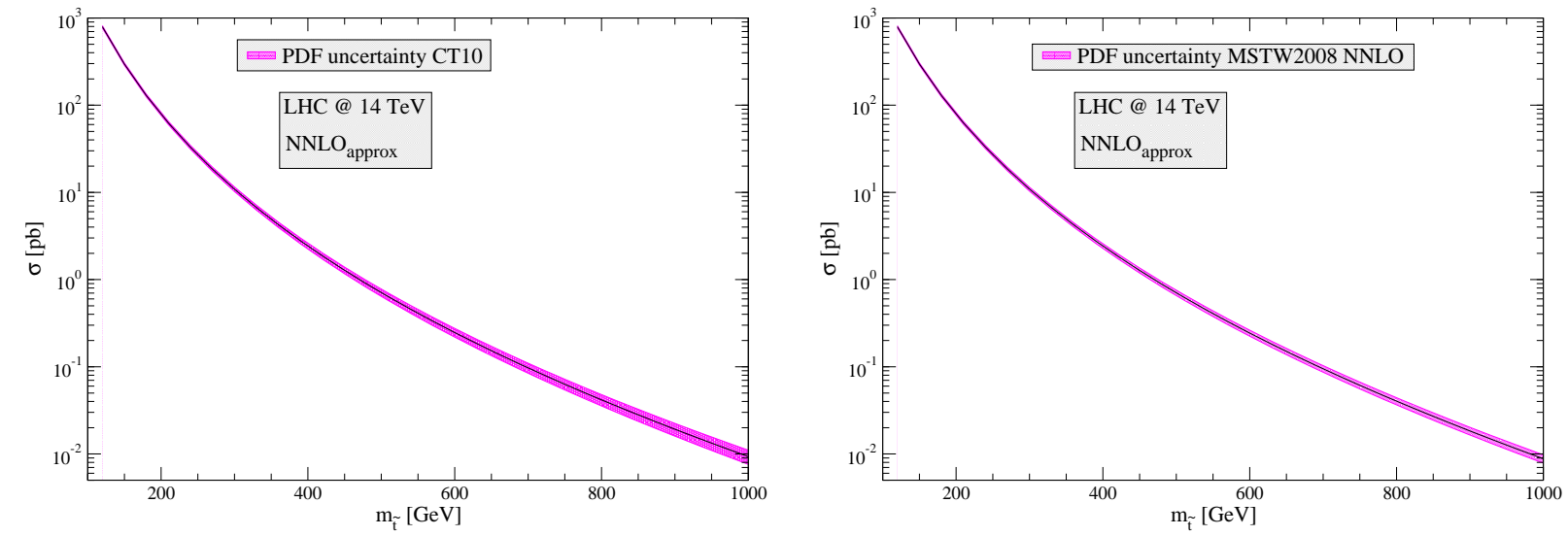

Figure 6: PDF uncertainty of the total NNLO cross section for the two PDF sets CT10 [41] (left figure) and MSTW2008 NNLO [39] (right figure) at the LHC (14 TeV). 
Combining theoretical uncertainty and PDF error one obtains

$$
\begin{aligned}
& \sigma_{\mathrm{NNLO}}=10.90 \mathrm{pb}{ }_{-0.18}^{+0.01} \mathrm{pb}(\text { scale }){ }_{-0.55}^{+0.55} \mathrm{pb} \text { (MSTW2008 NNLO) } \\
& \sigma_{\mathrm{NNLO}}=10.86 \mathrm{pb}{ }_{-0.19}^{+0.01} \mathrm{pb}(\text { scale }){ }_{-0.64}^{+0.65} \mathrm{pb} \text { (CT10). }
\end{aligned}
$$

\subsection{Mass exclusion limits}

The approximated NNLO contributions enlarge the stop-antistop production cross section by $\approx 10-20 \%$, depending on the hadron collider, its center of mass energy, and the stop mass. This could be converted into larger exclusion limits for the mass of the stop squark. A stop with a mass $m_{\tilde{t}_{1}}=120 \mathrm{GeV}$ has a NLO production cross section of $5.05 \mathrm{pb}$, the same cross section corresponds at NNLO to a stop with a mass of $123.5 \mathrm{GeV}$. At higher stop masses, the exclusion limit is even further enhanced: $0.164 \mathrm{pb}$ corresponds at NLO to a stop with a mass of $210 \mathrm{GeV}$, but at NNLO to a mass of $215 \mathrm{GeV}$. At the $\mathrm{LHC}(14 \mathrm{TeV})$ one would have a similar situation. An NLO cross section of $750 \mathrm{pb}$ corresponds to $m_{\tilde{t}_{1}}=120 \mathrm{GeV}$, but to $m_{\tilde{t}_{1}}=121.5 \mathrm{GeV}$ at NNLO. And if the stops are heavier, a cross section of $10 \mathrm{pb}$ is related to $m_{\tilde{t}_{1}}=300 \mathrm{GeV}$ at NLO, but to $m_{\tilde{t}_{1}}=305.5 \mathrm{GeV}$ at NNLO.

\section{Conclusion and Summary}

In this paper, I computed the NNLO threshold contributions including Coulomb corrections for stop-antistop production at hadron colliders.

- I presented analytical formulas for the threshold expansion of the NNLO scaling function using resummation techniques for the scale independent scaling function and RGE techniques for the scale-dependent scaling functions.

- After convolution with suitable PDF sets, the NNLO corrections are found to be about $20 \%$ for the Tevatron and $10-20 \%$ for the LHC compared to the hadronic NLO cross section. The PDF sets Cteq6.6 [40], MSTW 2008 NNLO [39], and CT10 [41] show only small differences in the total cross section, whereas the values obtained with the PDF set ABKM09 NNLO (5 flavours) [42] differ by $10-35 \%$ due to differences in the gluon PDF.

- I calculated the exact scale dependence and I found a remarkable stabilisation of the cross section under scale variation. For my example point, the theoretical error is reduced from $12 \%$ at NLO to better than $2 \%$ at NNLO.

- I discussed three types of errors: systematic theoretical errors, uncertainties due to scale variation and PDF errors. The systematic error was estimated to be about $3-6 \%$, the scale uncertainty to be about $2 \%$ or better, and the PDF error $3-18 \%$ depending on the stop mass and the PDF set used.

- Finally, I demonstrated how NNLO cross sections could enlarge exclusion limits. The improvement of the lower exclusion limit were about a few $\mathrm{GeV}$.

\section{Acknowledgments}

I would like to thank P. Uwer and A. Kulesza, W. Porod, and S. Uccirati for useful discussions and S. Moch and M. Krämer for reading the manuscript and giving helpful comments. This work 
is supported in part by the Helmholtz Alliance "Physics at the Terascale" (HA-101) and by the research training group GRK 1147 of the Deutsche Forschungsgemeinschaft.

\section{A Useful Formulas}

\section{A.1 Mellin Transformations}

$$
\begin{aligned}
& \int_{0}^{1} \mathrm{~d} \rho \rho^{N-1} \beta^{3} \ln \beta=-\frac{\sqrt{\pi}}{8}[-8+6 \ln 2+3 \ln \tilde{N}] \frac{1}{N^{5 / 2}}(1+\mathcal{O}(1 / N)) \\
& \int_{0}^{1} \mathrm{~d} \rho \rho^{N-1} \beta^{3} \ln ^{2} \beta=\frac{\sqrt{\pi}}{32}\left[3 \pi^{2}+16-64 \ln 2+24 \ln ^{2} 2+8(-4+3 \ln 2) \ln \tilde{N}+6 \ln ^{2} \widetilde{N}\right] \\
& \times \frac{1}{N^{5 / 2}}(1+\mathcal{O}(1 / N)) \\
& \int_{0}^{1} \mathrm{~d} \rho \rho^{N-1} \beta^{3} \ln ^{3} \beta=-\frac{3 \sqrt{\pi}}{64}\left[32 \ln 2-64 \ln ^{2} 2+16 \ln ^{3} 2-8 \pi^{2}+6 \pi^{2} \ln 2+28 \zeta_{3}\right. \\
& +\left(16+3 \pi^{2}-64 \ln 2+24 \ln ^{2} 2\right) \ln \tilde{N} \\
& \left.+2(-8+6 \ln 2) \ln ^{2} \tilde{N}+2 \ln ^{3} \tilde{N}\right] \frac{1}{N^{5 / 2}}(1+\mathcal{O}(1 / N)) \\
& \int_{0}^{1} \mathrm{~d} \rho \rho^{N-1} \beta^{3} \ln ^{4} \beta=\frac{\sqrt{\pi}}{256}\left[21 \pi^{4}+12 \pi^{2}\left(8-32 \ln 2+12 \ln ^{2} 2\right)\right. \\
& +4\left(192 \ln ^{2} 2-256 \ln ^{3} 2+48 \ln ^{4} 2-448 \zeta_{3}+336 \zeta_{3} \ln 2\right) \\
& +24\left(-8 \pi^{2}+6 \pi^{2} \ln 2+32 \ln 2-64 \ln ^{2} 2+16 \ln ^{3} 2+28 \zeta_{3}\right) \ln \tilde{N} \\
& +12\left(16+3 \pi^{2}-64 \ln 2+24 \ln ^{2} 2\right) \ln ^{2} \tilde{N} \\
& \left.+16(-8+6 \ln 2) \ln ^{3} \tilde{N}+12 \ln ^{4} \tilde{N}\right] \frac{1}{N^{5 / 2}}(1+\mathcal{O}(1 / N)) \\
& \int_{0}^{1} \mathrm{~d} \rho \rho^{N-1} \beta^{2} \ln \beta=-\frac{1}{2} \ln \widetilde{N} \frac{1}{N^{2}}(1+\mathcal{O}(1 / N)) \\
& \int_{0}^{1} \mathrm{~d} \rho \rho^{N-1} \beta^{2} \ln ^{2} \beta=\frac{1}{24}\left[\pi^{2}-12 \ln \tilde{N}+6 \ln ^{2} \tilde{N}\right] \frac{1}{N^{2}}(1+\mathcal{O}(1 / N))
\end{aligned}
$$

with $\ln \tilde{N}=\ln N+\gamma_{e}$. A detailed overview of Mellin transformations is given in Ref. [43]. 


\section{A.2 Resummation}

For the sake of clarity, I specify the function $g_{q \bar{q}, 8}^{0}$ I have used in my resummation. The function $g_{q \bar{q}, 8}^{0}$ differs a little bit from the function given in Ref. [21] due to Mellin transformation of functions of the type $\beta^{3} \log ^{k}(\beta)$ instead of $\beta \log ^{k}(\beta), 0 \leq k \leq 4$, as it is in the case of $t \bar{t}$-production.

$$
\begin{aligned}
& g_{q \bar{q}, 8}^{0}=1+\alpha_{s}\left[C_{F}\left(-64+8 \gamma_{e}^{2}+4 \pi^{2}-32 \ln 2+8 \ln ^{2} 2\right)+C_{A}\left(-8+4 \gamma_{e}-4 \ln 2\right)+4 C_{q \bar{q}}^{(1)}\right. \\
& \left.+2 C_{F}\left(16-8 \gamma_{e}\right) \ln 2\right] \\
& +\alpha_{s}^{2}\left[C_{F} C_{q \bar{q}}^{(1)}\left(-\frac{3328}{9}+32 \gamma_{e}^{2}+16 \pi^{2}+512 \ln 2-64 \gamma_{e} \ln 2-256 \ln ^{2} 2\right)\right. \\
& +C_{F} n_{f}\left(\frac{30592}{81}-\frac{448}{9} \zeta_{3}-\frac{224}{27} \gamma_{e}-\frac{80}{9} \gamma_{e}^{2}-\frac{32}{9} \gamma_{e}^{3}-\frac{56}{3} \pi^{2}-\frac{14272}{27} \ln 2\right. \\
& \left.+\frac{160}{9} \gamma_{e} \ln 2+\frac{32}{3} \gamma_{e}^{2} \ln 2+16 \pi^{2} \ln 2+\frac{2944}{9} \ln ^{2} 2-\frac{32}{3} \gamma_{e} \ln ^{2} 2-\frac{832}{9} \ln ^{3} 2\right) \\
& +C_{F}^{2}\left(-\frac{335872}{27}+\frac{14336}{3} \zeta_{3}-512 \gamma_{e}^{2}+32 \gamma_{e}^{4}+\frac{4352}{9} \pi^{2}+32 \pi^{2} \gamma_{e}^{2}+24 \pi^{4}\right. \\
& +\frac{57344}{3} \ln 2-5376 \zeta_{3} \ln 2+1024 \gamma_{e} \ln 2-128 \gamma_{e}^{3} \ln 2-1024 \pi^{2} \ln 2 \\
& -64 \pi^{2} \gamma_{e} \ln 2-15872 \ln ^{2} 2+192 \gamma_{e}^{2} \ln ^{2} 2+608 \pi^{2} \ln ^{2} 2+9216 \ln ^{3} 2 \\
& \left.-128 \gamma_{e} \ln ^{3} 2-2560 \ln ^{4} 2\right) \\
& +C_{A} C_{q \bar{q}}^{(1)}\left(-\frac{128}{3}+16 \gamma_{e}+32 \ln 2\right) \\
& +C_{A} n_{f}\left(\frac{512}{9}-\frac{88}{9} \gamma_{e}-\frac{8}{3} \gamma_{e}^{2}-\frac{4}{3} \pi^{2}-\frac{464}{9} \ln 2+\frac{16}{3} \gamma_{e} \ln 2+\frac{64}{3} \ln ^{2} 2\right) \\
& +C_{A} C_{F}\left(-\frac{250432}{81}+\frac{7840}{9} \zeta_{3}-\frac{5296}{27} \gamma_{e}-56 \gamma_{e} \zeta_{3}-\frac{40}{9} \gamma_{e}^{2}+\frac{464}{9} \gamma_{e}^{3}+\frac{5188}{27} \pi^{2}\right. \\
& +16 \pi^{2} \gamma_{e}-\frac{8}{3} \pi^{2} \gamma_{e}^{2}-\frac{4}{3} \pi^{4}+\frac{132256}{27} \ln 2-112 \zeta_{3} \ln 2+\frac{80}{9} \gamma_{e} \ln 2 \\
& -\frac{464}{3} \gamma_{e}^{2} \ln 2-\frac{728}{3} \pi^{2} \ln 2+\frac{16}{3} \pi^{2} \gamma_{e} \ln 2-\frac{33088}{9} \ln ^{2} 2+\frac{464}{3} \gamma_{e} \ln ^{2} 2 \\
& \left.+\frac{64}{3} \pi^{2} \ln ^{2} 2+\frac{12064}{9} \ln ^{3} 2\right) \\
& +C_{A}^{2}\left(-\frac{2816}{9}+\frac{172}{9} \gamma_{e}+\frac{68}{3} \gamma_{e}^{2}+\frac{134}{9} \pi^{2}-\frac{4}{3} \pi^{2} \gamma_{e}+\frac{3608}{9} \ln 2-\frac{136}{3} \gamma_{e} \ln 2\right. \\
& \left.-\frac{8}{3} \pi^{2} \ln 2-\frac{544}{3} \ln ^{2} 2-\frac{64}{3} \zeta_{3}+16 \zeta_{3} \ln 2+8 \gamma_{e} \zeta_{3}\right) \\
& \left.+C_{q \bar{q}}^{(2)}\right]
\end{aligned}
$$

$\gamma_{e}$ denotes the Euler constant and $\zeta_{3}$ is the Riemannian Zeta function $\zeta(x)$ evaluated at $x=3$. 


\section{B Numerical Results}

\begin{tabular}{|c|c|c|c|c|c|c|c|c|c|}
\hline \multirow{2}{*}{$\begin{array}{c}m_{\tilde{t}} \\
\mathrm{GeV}]\end{array}$} & \multicolumn{3}{|c|}{$\sigma(\mathrm{LO})[\mathrm{pb}]$} & \multicolumn{3}{|c|}{$\sigma(\mathrm{NLO})[\mathrm{pb}]$} & \multicolumn{3}{|c|}{$\sigma(\mathrm{NNLO})[\mathrm{pb}]$} \\
\hline & $x=\frac{1}{2}$ & $x=1$ & $x=2$ & $x=\frac{1}{2}$ & $x=1$ & $x=2$ & $x=\frac{1}{2}$ & $x=1$ & $x=2$ \\
\hline \multicolumn{10}{|c|}{ Cteq6.6 } \\
\hline 120 & 579.745 & 461.516 & 372.340 & 842.538 & 741.438 & 652.012 & 762.236 & 786.927 & 785.150 \\
\hline 180 & 97.170 & 75.718 & 60.001 & 133.421 & 118.340 & 103.980 & 124.929 & 127.242 & 126.175 \\
\hline 240 & 25.299 & 19.463 & 15.254 & 33.578 & 29.929 & 26.279 & 32.176 & 32.490 & 32.086 \\
\hline 300 & 8.466 & 6.455 & 5.020 & 10.976 & 9.817 & 8.615 & 10.702 & 10.739 & 10.573 \\
\hline 360 & 3.335 & 2.526 & 1.953 & 4.250 & 3.810 & 3.342 & 4.201 & 4.196 & 4.121 \\
\hline 480 & 0.710 & 0.532 & 0.408 & 0.883 & 0.794 & 0.696 & 0.892 & 0.884 & 0.865 \\
\hline 600 & 0.198 & 0.147 & 0.112 & 0.242 & 0.218 & 0.191 & 0.249 & 0.245 & 0.239 \\
\hline \multicolumn{10}{|c|}{ MSTW 2008 NNLO } \\
\hline 120 & 587.154 & 468.209 & 378.090 & 849.643 & 749.028 & 659.621 & 769.079 & 793.744 & 791.998 \\
\hline 180 & 99.033 & 77.177 & 61.136 & 135.263 & 120.146 & 105.649 & 126.755 & 129.046 & 127.921 \\
\hline 240 & 25.825 & 19.848 & 15.538 & 34.083 & 30.413 & 26.712 & 32.698 & 32.996 & 32.566 \\
\hline 300 & 8.632 & 6.571 & 5.101 & 11.127 & 9.960 & 8.740 & 10.867 & 10.896 & 10.718 \\
\hline 360 & 3.390 & 2.562 & 1.977 & 4.295 & 3.854 & 3.379 & 4.255 & 4.245 & 4.165 \\
\hline 480 & 0.714 & 0.534 & 0.408 & 0.883 & 0.795 & 0.696 & 0.895 & 0.886 & 0.866 \\
\hline 600 & 0.197 & 0.146 & 0.111 & 0.239 & 0.216 & 0.189 & 0.247 & 0.243 & 0.236 \\
\hline \multicolumn{10}{|c|}{ CT10 } \\
\hline 120 & 584.754 & 465.703 & 375.794 & 849.755 & 747.907 & 657.825 & 768.385 & 793.474 & 791.811 \\
\hline 180 & 98.265 & 76.560 & 60.641 & 134.841 & 119.609 & 105.071 & 126.243 & 128.588 & 127.483 \\
\hline 240 & 25.605 & 19.688 & 15.415 & 33.955 & 30.263 & 26.555 & 32.541 & 32.854 & 32.425 \\
\hline 300 & 8.568 & 6.528 & 5.070 & 11.098 & 9.923 & 8.699 & 10.824 & 10.858 & 10.679 \\
\hline 360 & 3.374 & 2.553 & 1.970 & 4.294 & 3.848 & 3.371 & 4.248 & 4.239 & 4.158 \\
\hline 480 & 0.717 & 0.537 & 0.410 & 0.890 & 0.800 & 0.700 & 0.900 & 0.891 & 0.870 \\
\hline 600 & 0.199 & 0.148 & 0.112 & 0.244 & 0.219 & 0.191 & 0.250 & 0.246 & 0.240 \\
\hline \multicolumn{10}{|c|}{ ABKM 09 NNLO (5 flv) } \\
\hline 120 & 539.054 & 428.594 & 345.556 & 764.660 & 677.764 & 598.338 & 700.513 & 720.046 & 718.084 \\
\hline 180 & 87.613 & 68.261 & 54.112 & 117.982 & 105.305 & 92.854 & 111.788 & 113.439 & 112.487 \\
\hline 240 & 22.132 & 17.046 & 13.380 & 28.906 & 25.912 & 22.833 & 28.027 & 28.207 & 27.868 \\
\hline 300 & 7.190 & 5.498 & 4.288 & 9.196 & 8.270 & 7.284 & 9.075 & 9.079 & 8.947 \\
\hline 360 & 2.752 & 2.093 & 1.626 & 3.464 & 3.123 & 2.751 & 3.468 & 3.454 & 3.397 \\
\hline 480 & 0.554 & 0.419 & 0.323 & 0.682 & 0.617 & 0.544 & 0.698 & 0.690 & 0.677 \\
\hline 600 & 0.146 & 0.110 & 0.085 & 0.177 & 0.161 & 0.142 & 0.185 & 0.182 & 0.178 \\
\hline
\end{tabular}

Table 3: Numerical values for the stop pair-production cross section at LHC with $\sqrt{s}=14 \mathrm{TeV}$ and the PDF sets Cteq6.6 [40], MSTW 2008 NNLO [39], ABKM09 NNLO (5 flavours) [42], and CT10 [41]. The QCD predictions are given at LO, NLO, and NNLO accuracy and for different stop masses and scales $x=\mu / m_{\tilde{t}}$. 


\begin{tabular}{|c|c|c|c|c|c|c|c|c|c|}
\hline \multirow{2}{*}{$\begin{array}{c}m_{\tilde{t}} \\
{[\mathrm{GeV}]}\end{array}$} & \multicolumn{3}{|c|}{$\sigma(\mathrm{LO})[\mathrm{pb}]$} & \multicolumn{3}{|c|}{$\sigma(\mathrm{NLO})[\mathrm{pb}]$} & \multicolumn{3}{|c|}{$\sigma(\mathrm{NNLO})[\mathrm{pb}]$} \\
\hline & $x=\frac{1}{2}$ & $x=1$ & $x=2$ & $x=\frac{1}{2}$ & $x=1$ & $x=2$ & $x=\frac{1}{2}$ & $x=1$ & $x=2$ \\
\hline \multicolumn{10}{|c|}{ Cteq6.6 } \\
\hline 120 & 134.391 & 101.197 & 77.851 & 184.136 & 160.840 & 138.709 & 173.624 & 176.078 & 173.026 \\
\hline 180 & 18.038 & 13.341 & 10.106 & 23.542 & 20.725 & 17.855 & 23.084 & 23.068 & 22.511 \\
\hline 240 & 3.881 & 2.839 & 2.130 & 4.925 & 4.354 & 3.748 & 4.957 & 4.907 & 4.765 \\
\hline 300 & 1.091 & 0.792 & 0.590 & 1.359 & 1.205 & 1.036 & 1.395 & 1.372 & 1.327 \\
\hline 360 & 0.364 & 0.263 & 0.195 & 0.448 & 0.398 & 0.342 & 0.468 & 0.457 & 0.441 \\
\hline 420 & 0.137 & 0.098 & 0.072 & 0.168 & 0.149 & 0.128 & 0.177 & 0.172 & 0.165 \\
\hline \multicolumn{10}{|c|}{ MSTW 2008 NNLO } \\
\hline 120 & 137.257 & 103.302 & 79.392 & 186.837 & 163.495 & 141.098 & 176.403 & 178.825 & 175.655 \\
\hline 180 & 18.377 & 13.561 & 10.249 & 23.825 & 20.998 & 18.086 & 23.416 & 23.378 & 22.789 \\
\hline 240 & 3.918 & 2.857 & 2.137 & 4.941 & 4.371 & 3.759 & 4.991 & 4.933 & 4.782 \\
\hline 300 & 1.087 & 0.786 & 0.584 & 1.346 & 1.194 & 1.025 & 1.388 & 1.362 & 1.315 \\
\hline 360 & 0.357 & 0.257 & 0.190 & 0.437 & 0.388 & 0.333 & 0.458 & 0.447 & 0.430 \\
\hline 420 & 0.132 & 0.094 & 0.069 & 0.160 & 0.142 & 0.122 & 0.170 & 0.165 & 0.159 \\
\hline \multicolumn{10}{|c|}{ CT10 } \\
\hline 120 & 136.074 & 102.422 & 78.752 & 186.261 & 162.710 & 140.298 & 175.639 & 178.121 & 175.005 \\
\hline 180 & 18.262 & 13.496 & 10.211 & 23.805 & 20.956 & 18.042 & 23.354 & 23.332 & 22.753 \\
\hline 240 & 3.922 & 2.866 & 2.146 & 4.971 & 4.394 & 3.777 & 5.008 & 4.955 & 4.805 \\
\hline 300 & 1.100 & 0.797 & 0.592 & 1.368 & 1.212 & 1.040 & 1.406 & 1.381 & 1.333 \\
\hline 360 & 0.366 & 0.263 & 0.195 & 0.450 & 0.399 & 0.342 & 0.470 & 0.459 & 0.441 \\
\hline 420 & 0.137 & 0.098 & 0.072 & 0.167 & 0.148 & 0.127 & 0.177 & 0.172 & 0.165 \\
\hline \multicolumn{10}{|c|}{ ABKM 09 NNLO (5 flv) } \\
\hline 120 & 117.501 & 88.531 & 68.185 & 157.745 & 138.823 & 120.224 & 151.054 & 152.485 & 149.896 \\
\hline 180 & 14.825 & 11.007 & 8.373 & 19.061 & 16.894 & 14.626 & 18.991 & 18.902 & 18.474 \\
\hline 240 & 3.004 & 2.215 & 1.674 & 3.765 & 3.353 & 2.904 & 3.856 & 3.804 & 3.704 \\
\hline 300 & 0.797 & 0.586 & 0.441 & 0.981 & 0.877 & 0.761 & 1.027 & 1.006 & 0.978 \\
\hline 360 & 0.252 & 0.185 & 0.139 & 0.306 & 0.274 & 0.238 & 0.326 & 0.318 & 0.308 \\
\hline 420 & 0.090 & 0.066 & 0.049 & 0.108 & 0.097 & 0.084 & 0.117 & 0.113 & 0.110 \\
\hline
\end{tabular}

Table 4: Numerical values for the stop pair-production cross section at LHC with $\sqrt{s}=7 \mathrm{TeV}$ and the same PDF sets as in Tab. 3 The QCD predictions are given at LO, NLO, and NNLO accuracy and for different stop masses and scales $x=\mu / m_{\tilde{t}}$. 


\begin{tabular}{|c|c|c|c|c|c|c|c|c|c|}
\hline \multirow{2}{*}{$\begin{array}{c}m_{\tilde{t}} \\
{[\mathrm{GeV}]}\end{array}$} & \multicolumn{3}{|c|}{$\sigma(\mathrm{LO})[\mathrm{pb}]$} & \multicolumn{3}{|c|}{$\sigma(\mathrm{NLO})[\mathrm{pb}]$} & \multicolumn{3}{|c|}{$\sigma(\mathrm{NNLO})[\mathrm{pb}]$} \\
\hline & $x=\frac{1}{2}$ & $x=1$ & $x=2$ & $x=\frac{1}{2}$ & & $x=2$ & $x=\frac{1}{2}$ & & $x=2$ \\
\hline \multicolumn{10}{|c|}{ Cteq6.6 } \\
\hline 120 & $\overline{525}$ & 3.850 & 79 & 5.782 & 5.246 & 509 & 3 & 6.144 & 5.780 \\
\hline 150 & 1.570 & 1.095 & 0.790 & 1.572 & 1.448 & 1.254 & .793 & 1.695 & 1.589 \\
\hline 180 & 0.525 & 0.366 & 0.264 & 0.519 & 0.480 & 0.417 & 0.597 & 0.562 & 0.524 \\
\hline 210 & 0.194 & 0.135 & 0.097 & 0.192 & 0.178 & 0.154 & 0.222 & 0.208 & 0.193 \\
\hline 240 & 0.076 & 0.053 & 0.038 & 0.076 & 0.070 & 0.061 & 0.088 & 0.082 & 0.076 \\
\hline 270 & 0.031 & 0.021 & 0.015 & 0.032 & 0.029 & 0.025 & 0.036 & 0.034 & 0.031 \\
\hline 300 & 0.013 & 0.009 & 0.006 & 0.013 & 0.012 & 0.010 & 0.015 & 0.014 & 0.013 \\
\hline \multicolumn{10}{|c|}{ MSTW 2008 NNLO } \\
\hline 120 & 5.336 & 3.712 & 2.677 & 5.562 & 5.047 & 4.336 & 6.242 & 5.930 & 5.568 \\
\hline 150 & 1.492 & 1.040 & 0.751 & 1.482 & 1.368 & 1.186 & 1.701 & 1.605 & 1.503 \\
\hline 180 & 0.493 & 0.344 & 0.248 & 0.482 & 0.448 & 0.390 & 0.558 & 0.524 & 0.489 \\
\hline 210 & 0.180 & 0.126 & 0.090 & 0.177 & 0.164 & 0.143 & 0.205 & 0.191 & 0.178 \\
\hline 240 & 0.070 & 0.049 & 0.035 & 0.069 & 0.064 & 0.056 & 0.080 & 0.075 & 0.069 \\
\hline 270 & 0.028 & 0.020 & 0.014 & 0.029 & 0.026 & 0.023 & 0.033 & 0.031 & 0.028 \\
\hline 300 & 0.012 & 0.008 & 0.006 & 0.012 & 0.011 & 0.009 & 0.014 & 0.013 & 0.012 \\
\hline \multicolumn{10}{|c|}{ CT10 } \\
\hline 120 & 5.546 & 3.861 & 2.784 & 5.793 & 5.256 & 4.516 & 6.479 & 6.164 & 5.795 \\
\hline 150 & 1.570 & 1.094 & 0.789 & 1.567 & 1.444 & 1.251 & 1.792 & 1.693 & 1.586 \\
\hline 180 & 0.524 & 0.365 & 0.263 & 0.515 & 0.478 & 0.415 & 0.595 & 0.559 & 0.521 \\
\hline 210 & 0.193 & 0.134 & 0.096 & 0.190 & 0.176 & 0.153 & 0.220 & 0.206 & 0.191 \\
\hline 240 & 0.075 & 0.052 & 0.037 & 0.075 & 0.070 & 0.060 & 0.087 & 0.081 & 0.075 \\
\hline 270 & 0.031 & 0.021 & 0.015 & 0.031 & 0.029 & 0.025 & 0.036 & 0.033 & 0.031 \\
\hline 300 & 0.013 & 0.009 & 0.006 & 0.013 & 0.012 & 0.010 & 0.015 & 0.014 & 0.013 \\
\hline \multicolumn{10}{|c|}{ ABKM 09 NNLO (5 flv) } \\
\hline 120 & 4.478 & 3.207 & 2.371 & 4.374 & 4.095 & 3.617 & 5.023 & 4.773 & 4.532 \\
\hline 150 & 1.312 & 0.942 & 0.698 & 1.222 & 1.165 & 1.040 & 1.423 & 1.348 & 1.278 \\
\hline 180 & 0.453 & 0.325 & 0.240 & 0.419 & 0.401 & 0.359 & 0.488 & 0.461 & 0.435 \\
\hline 210 & 0.172 & 0.123 & 0.090 & 0.161 & 0.153 & 0.137 & 0.186 & 0.175 & 0.165 \\
\hline 240 & 0.069 & 0.049 & 0.036 & 0.066 & 0.062 & 0.055 & 0.076 & 0.071 & 0.067 \\
\hline 270 & 0.028 & 0.020 & 0.015 & 0.028 & 0.026 & 0.023 & 0.032 & 0.030 & 0.028 \\
\hline 300 & 0.012 & 0.008 & 0.006 & 0.012 & 0.011 & 0.010 & 0.014 & 0.013 & 0.012 \\
\hline
\end{tabular}

Table 5: Numerical values for the stop pair-production cross section at the Tevatron with $\sqrt{s}=1.96 \mathrm{TeV}$ and the same PDF sets as in Tab. 3. The QCD predictions are given at LO, NLO, and NNLO accuracy and for different stop masses and scales $x=\mu / m_{\tilde{t}}$. 


\section{References}

[1] Howard E. Haber and Gordon L. Kane. The Search for Supersymmetry: Probing Physics Beyond the Standard Model. Phys.Rept., 117:75-263, 1985.

[2] Hans Peter Nilles. Supersymmetry, Supergravity and Particle Physics. Phys.Rept., 110:1-162, 1984.

[3] I.J.R. Aitchison. Supersymmetry in Particle Physics. An Elementary Introduction. 2007.

[4] Gordon L. Kane, Alexey A. Petrov, Jing Shao, and Lian-Tao Wang. Initial determination of the spins of the gluino and squarks at LHC. J.Phys.G, G37:045004, 2010, arXiv:0805.1397.

[5] W. Beenakker, M. Krämer, T. Plehn, M. Spira, and P. M. Zerwas. Stop production at hadron colliders. Nucl. Phys., B515:3-14, 1998, hep-ph/9710451.

[6] T. Aaltonen et al. Search for Direct Pair Production of Supersymmetric Top and Supersymmetric Bottom Quarks in $p$ anti-p Collisions at $s^{* *}(1 / 2)=1.96-\mathrm{TeV}$. Phys.Rev., D76:072010, 2007, arXiv:0707.2567.

[7] Andrew G. Ivanov. Search for Pair Production of Supersymmetric Top Quarks Mimicking Standard Model Top Event Signatures at CDF. 2008, arXiv:0811.0788.

[8] T. Aaltonen et al. Search for Pair Production of Supersymmetric Top Quarks in Dilepton Events from $\mathrm{p}$ anti-p Collisions at $\mathrm{S}^{* *}(1 / 2)=1.96 \mathrm{TeV}$. Phys.Rev.Lett., 104:251801, 2010, arXiv:0912.1308.

[9] T. Aaltonen et al. Search for the supersymmetric partner of the top quark in $p \bar{p}$ collisions at $\sqrt{s}=1.96$ TeV. Phys.Rev.D, 2010, arXiv:1009.0266.

[10] V. M. Abazov et al. Search for admixture of scalar top quarks in the $t \bar{t}$ lepton+jets final state at $\sqrt{s}=1.96 \mathrm{TeV}$. Phys. Lett., B674:4-10, 2009, arXiv:0901.1063.

[11] V. M. Abazov et al. Search for the lightest scalar top quark in events with two leptons in $p \bar{p}$ collisions at $\sqrt{s}=1.96-$ TeV. Phys. Lett., B675:289, 2009, arXiv:0811.0459.

[12] W. Beenakker, R. Höpker, M. Spira, and P. M. Zerwas. Squark and gluino production at hadron colliders. Nucl. Phys., B492:51-103, 1997, hep-ph/9610490.

[13] A. Kulesza and L. Motyka. Threshold resummation for squark-antisquark and gluino- pair production at the LHC. Phys. Rev. Lett., 102:111802, 2009, arXiv:0807.2405.

[14] Ulrich Langenfeld and Sven-Olaf Moch. Higher-order soft corrections to squark hadro- production. Phys. Lett., B675:210-221, 2009, arXiv:0901.0802.

[15] Wim Beenakker, Silja Brensing, Michael Krämer, Anna Kulesza, Eric Laenen, et al. Soft-gluon resummation for squark and gluino hadroproduction. JHEP, 0912:041, 2009, 0909.4418.

[16] M. Beneke, P. Falgari, and C. Schwinn. Soft and Coulomb gluon resummation in squarkantisquark production at the LHC. PoS, RADCOR2009:012, 2010, 1001.4627.

[17] Wolfgang Hollik, Monika Kollar, and Maike K. Trenkel. Hadronic production of top-squark pairs with electroweak NLO contributions. JHEP, 0802:018, 2008, 0712.0287. 
[18] M. Beccaria, G. Macorini, L. Panizzi, F.M. Renard, and C. Verzegnassi. Stop-antistop and sbottom-antisbottom production at LHC: A One-loop search for model parameters dependence. Int.J.Mod.Phys., A23:4779-4810, 2008, 0804.1252.

[19] Wim Beenakker et al. Supersymmetric top and bottom squark production at hadron colliders. 2010, arXiv:1006.4771.

[20] W. Beenakker, R. Höpker, and M. Spira. PROSPINO: A program for the PROduction of Supersymmetric Particles In Next-to-leading Order QCD. 1996, hep-ph/9611232.

[21] Sven Moch and Peter Uwer. Theoretical status and prospects for top-quark pair production at hadron colliders. Phys. Rev., D78:034003, 2008, arXiv:0804.1476.

[22] U. Langenfeld, S. Moch, and P. Uwer. Measuring the running top-quark mass. Phys. Rev., D80:054009, 2009, 0906.5273.

[23] U. Langenfeld. Squark pair production at the LHC. AIP Conf. Proc., 1200:333-336, 2010, arXiv:0908.3567.

[24] Harry Contopanagos, Eric Laenen, and George F. Sterman. Sudakov factorization and resummation. Nucl. Phys., B484:303-330, 1997, hep-ph/9604313.

[25] Stefano Catani, Michelangelo L. Mangano, Paolo Nason, and Luca Trentadue. The Resummation of Soft Gluon in Hadronic Collisions. Nucl. Phys., B478:273-310, 1996, hep-ph/9604351.

[26] Nikolaos Kidonakis and George F. Sterman. Resummation for QCD hard scattering. Nucl. Phys., B505:321-348, 1997, hep-ph/9705234.

[27] S. Moch, J. A. M. Vermaseren, and A. Vogt. Higher-order corrections in threshold resummation. Nucl. Phys., B726:317-335, 2005, hep-ph/0506288.

[28] Michal Czakon, Alexander Mitov, and George F. Sterman. Threshold Resummation for Top-Pair Hadroproduction to Next-to-Next-to-Leading Log. Phys.Rev., D80:074017, 2009, arXiv:0907.1790.

[29] Martin Beneke, Michal Czakon, Pietro Falgari, Alexander Mitov, and Christian Schwinn. Threshold expansion of the $g g(q \bar{q}) \rightarrow Q \bar{Q}+X$ cross section at $\mathcal{O}\left(\alpha_{s}^{4}\right)$. Phys.Lett., B690:483490, 2010, arXiv:0911.5166.

[30] S. Moch, J. A. M. Vermaseren, and A. Vogt. The three-loop splitting functions in QCD: The non-singlet case. Nucl. Phys., B688:101-134, 2004, hep-ph/0403192.

[31] J. Kodaira and L. Trentadue. Summing Soft Emission in QCD. Phys. Lett., B112:66-70, 1982.

[32] M. Beneke, P. Falgari, and C. Schwinn. Soft radiation in heavy-particle pair production: Allorder colour structure and two-loop anomalous dimension. Nucl.Phys., B828:69-101, 2010, arXiv:0907.1443.

[33] S. Moch and A. Vogt. On non-singlet physical evolution kernels and large-x coefficient functions in perturbative QCD. JHEP, 0911:099, 2009, arXiv:0909.2124.

[34] Eric Laenen, Gerben Stavenga, and Chris D. White. Path integral approach to eikonal and next-to-eikonal exponentiation. JHEP, 0903:054, 2009, arXiv:0811.2067. 
[35] Andrzej Czarnecki and Kirill Melnikov. Two loop QCD corrections to the heavy quark pair production cross-section in e+ e- annihilation near the threshold. Phys.Rev.Lett., 80:2531-2534, 1998, hep-ph/9712222.

[36] W. L. van Neerven and A. Vogt. NNLO evolution of deep-inelastic structure functions: The singlet case. Nucl. Phys., B588:345-373, 2000, hep-ph/0006154.

[37] Nikolaos Kidonakis, Eric Laenen, Sven Moch, and Ramona Vogt. Sudakov resummation and finite order expansions of heavy quark hadroproduction cross sections. Phys. Rev., D64:114001, 2001, hep-ph/0105041.

[38] A. Vogt, S. Moch, and J. A. M. Vermaseren. The three-loop splitting functions in QCD: The singlet case. Nucl. Phys., B691:129-181, 2004, hep-ph/0404111.

[39] A.D. Martin, W.J. Stirling, R.S. Thorne, and G. Watt. Parton distributions for the LHC. Eur.Phys.J., C63:189-285, 2009, arXiv:0901.0002.

[40] Pavel M. Nadolsky et al. Implications of CTEQ global analysis for collider observables. Phys. Rev., D78:013004, 2008, arXiv:0802.0007.

[41] Hung-Liang Lai, Marco Guzzi, Joey Huston, Zhao Li, Pavel M. Nadolsky, et al. New parton distributions for collider physics. 2010, arXiv:1007.2241.

[42] S. Alekhin, J. Blümlein, S. Klein, and S. Moch. The 3, 4, and 5-flavor NNLO Parton from Deep-Inelastic-Scattering Data and at Hadron Colliders. Phys.Rev., D81:014032, 2010, arXiv:0908.2766.

[43] J. Blümlein. Structural Relations of Harmonic Sums and Mellin Transforms up to Weight w = 5. Comput.Phys.Commun., 180:2218-2249, 2009, arXiv:0901.3106. 